\title{
DRUG UTILIZATION PATTERN IN PREGNANCY AMONGST INPATIENT AND OUTPATIENT GROUPS IN A SPECIALIST HOSPITAL, NORTH-WESTERN NIGERIA
}

\author{
IYABO ADEBISI, TIJJANI RABIU GIAZE \\ Department of Pharmacology and Toxicology, Usmanu Dan-Fodiyo University, Sokoto-Nigeria \\ Email: rtgiaze1143@gmail.com
}

Received:29 Apr 2016 Revised and Accepted: 17 Jan 2017

\begin{abstract}
Objective: Drugs prescribed in pregnancy may diffuse across the placenta to the fetus thereby posing a great risk of teratogenicity. In this study, drug utilization pattern in pregnancy amongst inpatient and outpatient groups was studied retrospectively in a specialist hospital in the North Western region of Nigerian.

Methods: Drug prescription information was collected from case notes of 2634 patients that attended the antenatal clinic on an outpatient basis and those that were admitted due to complication(s) for a period of six months. Data collected were analyzed using Microsoft excel software and compared with FDA pregnancy categorization of drugs.

Results: In the outpatient group, 215 patients had complaints that warranted the use of prescription drugs while in the inpatient group, 69 patients had complications that necessitated hospitalisation. In the outpatient group, prescribed drugs for the treatment of malaria (34\%) and urinary tract infection (25\%) were predominantly from FDA category B. In the inpatient group, drugs for malaria (18\%), anemia (11.6\%) and pre-eclampsia $(11.6 \%)$ were the most prescribed. In the management of anemia, drugs prescribed were purely from FDA category A, while, in the case of preeclampsia, a significant amount of prescribed drugs were from FDA categories $\mathrm{C}$ and $\mathrm{N}$.
\end{abstract}

Conclusion: In both patient groups, no drug from FDA category X was prescribed. Therefore, it can be concluded that drugs prescribed in pregnancy in specialist hospital, Sokoto-Nigeria, were within a reasonable limit of safety. However, the use of drugs such as diazepam whose risks are yet to be classified should be avoided.

Keywords: Pregnancy, Teratogenicity, Pre-eclampsia, Drug utilization, Sokoto specialist

(c) 2016 The Authors. Published by Innovare Academic Sciences Pvt Ltd. This is an open access article under the CC BY license (http://creativecommons.org/licenses/by/4. 0/) DOI: http://dx.doi.org/10.22159/ijpps.2017v9i3.12486

\section{INTRODUCTION}

Regardless of the limited information on safety, the use of drugs in pregnancy is common in medical practice [1]. Drugs are commonly prescribed in pregnancy for conditions of deficiency directly related to pregnancy such as supplementation with drugs like iron, folic acid, calcium, and vitamins in the management of anemia, or for the treatment of conditions secondary to pregnancy such as urinary tract infection, eclampsia and many more.

Drugs are also used in pregnancy to treat pre-existing chronic conditions such as diabetes, epilepsy and hypertension [2]. Due to placental transport of maternal substrate to the fetus, substances of low molecular weight including drugs can diffuse freely across the placenta to fetus [3]. Therefore, the use of drugs in pregnancy is an issue of great concern to the patients as well as prescribers [2]. This assertion is proven true by previous incidences of teratogenicity in the past, for example, the thalidomide and diethylstilbestrol incidences of 1960 and 1971, respectively. Also, it has been reported that medicines are responsible for $1 \%$ of reported cases of congenital malformations [4].

Often, due to ethical reasons, manufacturers of pharmaceuticals almost never screen new drugs for safety in pregnant women. Moreover, results of safety from the animal study are not always extrapolated in human population. These constraints often compel manufacturers of pharmaceuticals to label most drugs as 'contraindicated in pregnancy' or 'use in pregnancy is not recommended unless potential benefits outweigh potential risk to fetuses' [5].

In view of this challenge, many regulatory bodies across the world have classified drugs into various pregnancy categories based on available evidence of risks or harmful effects associated with their use in pregnancy. Notable amongst these are Food and Drug Administration categorization [6]; Australian categorization; German categorization and many more. These categorizations are aimed at guiding health professions in the selection of prescription drugs for use in pregnancy.
Drugs used in pregnancy, in the FDA and German categorization, are grouped based on their hierarchy of risk with increasing order of risk down the table while Australian categorization is independent on the hierarchy of risk to cause defects to fetuses.

However, it is recommended that drugs that have been in use for a long period of time should be encouraged to be prescribed during pregnancy at possibly lower end of therapeutic dose range.

This research work is an investigation into drug utilization pattern in pregnancy amongst inpatient and outpatient groups at the Obstetrics and Gynecology Department of a specialist hospital in North Western Nigeria. It aims at creating useful research data that will promote safe and effective use of medicines in pregnancy by health practitioners in North Western Nigeria and thereby improving overall health outcome. It is aimed at putting together information on drug prescription practices and prevalent complaints that warrant the use of the $\operatorname{drug}(\mathrm{s})$ in pregnancy from in and out patients' case folders and evaluating the degree of adherence of these prescriptions to standard recommendations by FDA.

\section{MATERIALS AND METHODS}

This study, retrospective in nature, was carried out at the antenatal clinic (ANC) of the Obstetrics and Gynecology Department of Specialist Hospital, Sokoto-Nigeria. It covers a period of 6 mo (January to June 2008). Drug prescription information was collected from case notes of pregnant women that attended the antenatal clinic on an outpatient basis (outpatient group) and those that were eventually admitted in the hospital as a result of complications (inpatient group). A total of 2634 folders were reviewed for the ANC during the study period. 215 patients had complaints that warranted the use of prescription drugs while 69 were admitted due to complaints of complications relating to pregnancy. The drugs used in both groups were classified according to diagnosis and evaluated in comparison with FDA categorization. The prevalent 
Table 1: FDA pregnancy categorization of medicines

\begin{tabular}{|c|c|c|}
\hline Category & Description & Examples \\
\hline A & Adequate and well-controlled studies have failed to demonstrate a risk to the fetus & $\begin{array}{l}\text { Levothyroxine, folic acid, magnesium } \\
\text { sulfate, liothyronine }\end{array}$ \\
\hline B & $\begin{array}{l}\text { Animal reproduction studies have failed to demonstrate a risk to the fetus and there are no } \\
\text { adequate and well-controlled studies in pregnant women. }\end{array}$ & $\begin{array}{l}\text { Levothyroxine, folic acid, magnesium } \\
\text { sulfate, liothyronine }\end{array}$ \\
\hline $\mathrm{C}$ & $\begin{array}{l}\text { Animal reproduction studies have shown an adverse effect on the fetus and no adequate studies } \\
\text { in humans, but the potential benefit may warrant use in pregnancy despite potential risks. }\end{array}$ & $\begin{array}{l}\text { Tramadol, gabapentin, amlodipine, } \\
\text { trazodone, prednisolone }\end{array}$ \\
\hline $\mathrm{D}$ & $\begin{array}{l}\text { There is positive evidence of human fetal risk, but the potential benefit may warrant use in } \\
\text { pregnancy despite potential risks. }\end{array}$ & $\begin{array}{l}\text { Lisinopril, alprazolam, losar-tan, } \\
\text { clonazepam, lorazepam }\end{array}$ \\
\hline $\mathrm{X}$ & $\begin{array}{l}\text { Positive fetal abnormalities and risks involved in the use of the drug in pregnant women } \\
\text { clearly outweigh potential benefits. }\end{array}$ & $\begin{array}{l}\text { Atorvastatin, simvastatin, warfarin, } \\
\text { methotrexate }\end{array}$ \\
\hline $\mathrm{N}$ & FDA has not yet classified the drug into a specified pregnancy category. & $\begin{array}{l}\text { Aspirin, oxycodone, acetaminophen, } \\
\text { diazepam }\end{array}$ \\
\hline
\end{tabular}

FDA = United States Food and Drug Administration and Control

\section{Ethics committee approval}

The Institutional Ethics Committee permission was taken prior to the commencement of the study.

\section{Hospital background}

The study was conducted at the Department of Obstetrics and Gynecology of the Specialist Hospital, Sokoto-Nigeria. The hospital is a 600-bed tertiary care hospital, which receives referrals from other private clinics, hospitals and general Physicians. It comprises of 13 departments under the stewardship of an experienced Medical Director.

\section{Prescription practices}

Pregnant women who attended the ANC were examined by a team of general practicing, consultant physicians and house surgeons of the department, and were given hand-written prescriptions. Pregnant women obtained their drugs either from hospital pharmacy or registered pharmacies outside the hospital when they are out-ofstock in the hospital. There was no facility to maintain pregnant women's health records electronically in the hospital.

\section{Inclusion criteria}

All pregnant patients greater than or equal to 11yrs of age were enrolled, via convenience sampling, in any trimester. All patients attending antenatal outpatient clinics or on admission in the ward, with or without co-morbidities and within the period of study were included in the study.

\section{Exclusion criteria}

Poorly legible prescriptions, without signature and date, pregnant women diagnosed with acute and chronic medical conditions not secondary to pregnancy that require hospitalization were excluded from the study.

\section{Statistical analysis}

Data collected were analyzed and presented as graphs using descriptive statistics with the help of Excel software, Microsoft office 2007.

\section{RESULTS}

At the end of this study, a total number of 2634 cases of pregnancy were recorded. $215(8.2 \%)$ outpatients and $69(2.6 \%)$ inpatients presented with conditions that necessitated prescription of drugs. In both groups, age group 21-30 presented the highest number of cases with prescription (fig. 1).

Malaria was the most common cause of prescription constituting 18.8 and $34.4 \%$ of the overall prescriptions in the inpatient and outpatient groups, respectively (fig. 2 and 3). In the inpatient group, in line with United States FDA categorization, $86 \%$ and $14 \%$ of prescribed medicines for malaria were from categories B and C, respectively, while 70 and $30 \%$ of medicines prescribed for threatened abortion were from categories B and N, respectively.

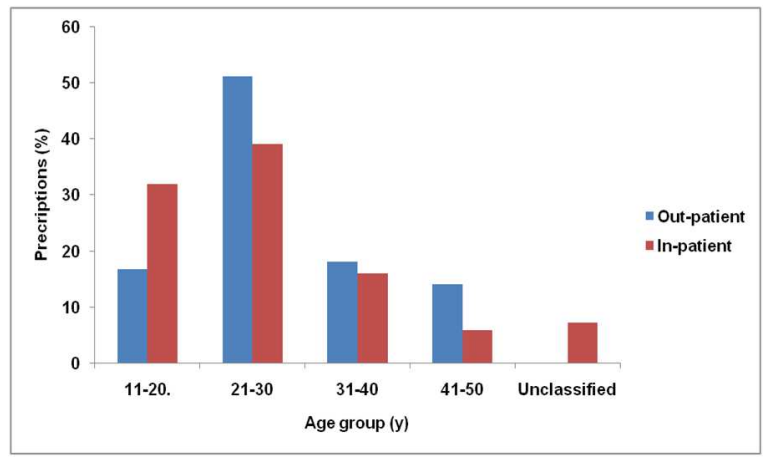

Fig. 1: Frequencies of pregnancy in different age groups amongst inpatient and outpatient groups

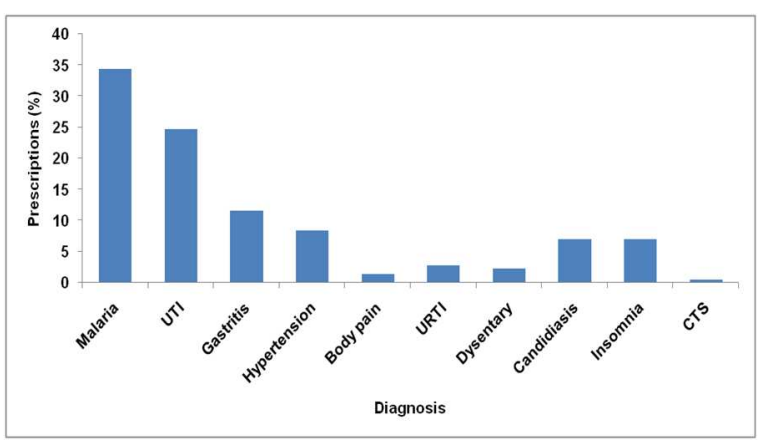

Fig. 2: Frequencies of prescription according to diagnosis amongst outpatient group, UTI; urinary tract infection, URTI; upper respiratory tract infection, CTS; Carpel Tunnel's syndrome

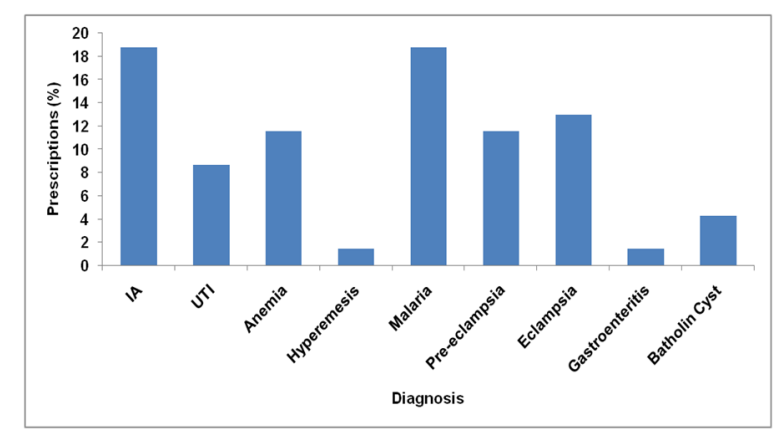

Fig. 3: Frequencies of prescription according to diagnosis amongst inpatient group, IA; incomplete abortion, UTI; urinary tract infection 
For UTI, 42.9 and 57.1 of drugs prescribed were grouped into categories B and C, respectively, while for severe anemia, $100 \%$ of prescribed drugs were from category A. For Pre-eclampsia, 84.21 and $15.79 \%$ of prescribed drugs belong to category $\mathrm{C}$ and $\mathrm{N}$, respectively. For eclampsia, 18.8\%, 16.6\%, $45.8 \%$ and $18.8 \%$ of medicines prescribed were from categories A, B, C and $\mathrm{N}$, respectively. For gastroenteritis, equal amount $(50 \%)$ of prescribed medicines were drawn from categories B and C (fig. 4).

In the outpatient group, $82.4 \%$ and $17.6 \%$ of prescribed medicines for malaria were from categories B and C, respectively while $86.6 \%$ and $13.4 \%$ medicines prescribed for candidiasis were from categories B and C, respectively. 86.8 and $13.2 \%$ of medicines prescribed for UTI were from categories B and C, respectively. For gastritis, $100 \%$ of prescribed medicines were from category B while 72.2 and $27.8 \%$ of prescribed medicines for hypertension were from categories B and C, respectively. 66.7 and $33.3 \%$ of prescriptions for body ache were from categories B and C, respectively. 83.4 and $16.6 \%$ of medicines prescribed for URTI were from categories B and C, respectively while 33.4 and $66.6 \%$ of medicines for dysentery were from categories B and $\mathrm{C}$, respectively. For insomnia, 66.7 and $33.3 \%$ of the medicines prescribed were from categories $\mathrm{B}$ and $\mathrm{N}$, respectively while in the case of carpel Tunnel's syndrome, prescribed medicines were equally distributed between categories A and B (fig. 5).

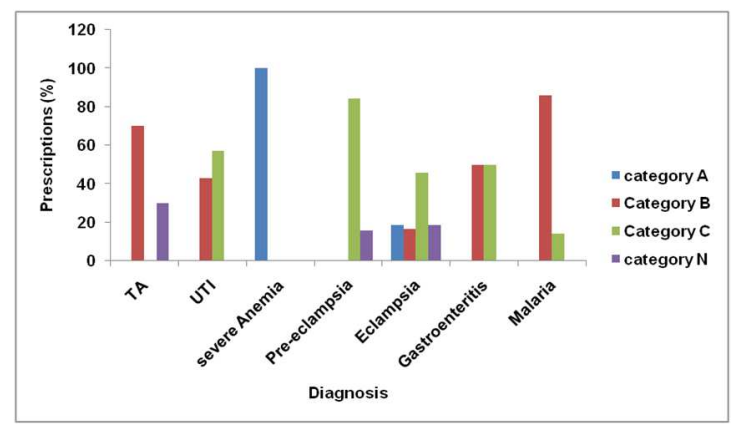

Fig. 4: Percentage prescription according to FDA categories amongst inpatient group, TA; Threatened abortion, UTI; urinary tract infection

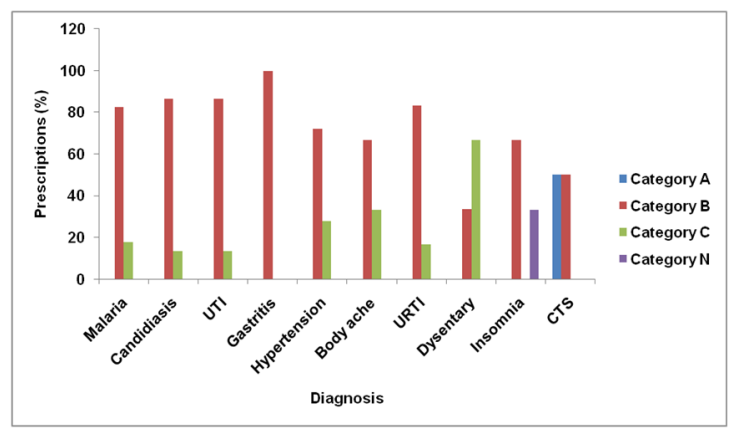

Fig. 5: Percentage prescription according to FDA categories amongst outpatient group, UTI; urinary tract infection, URTI; upper respiratory tract infection, CTS; Carpel-Tunnel's syndrome

\section{DISCUSSION}

In as much as drugs play an important role in improving and promoting the overall well-being of Mankind, it is expected that they are safe when used [4]. Previous studies carried out by WHO [7], Tsega et al. [8], Abubakar et al. [9], Ilyaz et al. [10] and many more researchers have shown that drugs are commonly used in pregnancy for the management of different medical conditions. Because the health of both the mother and fetus is at stake, drug use in pregnancy requires special consideration of safety in addition to efficacy [8]. At the end of this study, it was revealed that the nature of diagnosis that warrants the use of drugs in pregnancy varies between inpatient and outpatient groups. Malaria, candidiasis, UTI, gastritis, hypertension, body ache, URTI, dysentery, insomnia and CTS were found to be the common medical conditions that necessitated the use of medicines in the outpatient group while severe malaria, threatened abortion, severe anemia, preeclampsia, eclampsia, Batholin cyst and gastroenteritis were the diagnosis that necessitated hospitalization and use of drugs in the inpatient group. In the outpatient group, 215 (8.2\%) patients received prescriptions while in the inpatient group only $69(2.6 \%)$ of patients received medication. This discrepancy could be accounted for by the nature of diagnosis in the inpatient group, as it requires a specialized choice of drugs on the basis of safety and efficacy [11]. In the outpatient group, with the exception of supplement such as folic acid, iron and vitamins, the commonly prescribed drugs were for malaria (34.4\%) and UTI $(24.7 \%)$. Contrarily, in the inpatient group, other than malaria medication (18.8\%), medication for severe anemia and preeclampsia ( $11.6 \%$ each) were also significantly prescribed. Drugs for the management of incomplete abortion also constituted $18.8 \%$ of prescriptions in this group. Because in this case, the fetuses were already lost, it was excluded from the analysis. Thus, antimalarial drugs constituted the most frequently prescribed drugs in both inpatient and outpatient groups. This could be partly accounted for by the fact that, in Sub-Saharan Africa, the prevalence of parasitic diseases such as malaria is high due to poor sanitary condition and control [12]. Chloroquine, artesunate and pyrimethamine-sulphadoxine combination (fansidar) were the most prescribed antimalarial drugs. These drugs belong to FDA category B and are known for their attributes of safety in pregnancy. On the ground of resistance of Plasmodium falciparum malaria to chemotherapeutic agents, the Federal Ministry of Health of Nigeria has withdrawn the use of chloroquine as a first line treatment for both complicated and uncomplicated malaria. However, it is still widely prescribed by many practitioners in Nigeria on the ground that significant treatment success is still achievable with Chloroquine. In the treatment of UTI, amoxicillin (50.9\%) and metronidazole (34\%) were the most prescribed antibiotics in both inpatient and outpatient groups while, in the treatment of severe anemia, ferrotone (iron, vit. $\mathrm{B}_{12}$ and $\mathrm{C}$ ) and folic acid were the most prescribed drugs. These drugs belong to FDA category A. In the treatment of pre-eclampsia, eclampsia and threatened abortion, diazepam constituted $15.8 \%, 18.8 \%$ and $30 \%$ prescribed drugs, respectively. These percentages were significantly high as diazepam is yet to be classified into any risk category (category $\mathrm{N})$ and is, therefore, likely to pose a higher risk to the fetus.

Summarily, except in the treatment of CTS in which $50 \%$ of prescribed drugs were from FDA category A, prescribed drugs in the outpatient group were mainly from categories B and C. FDA Category $\mathrm{A}$ is known to be constituted of drugs in which adequate and well-controlled studies have failed to demonstrate a risk to the fetus while category B comprises of drugs in which animal reproduction studies have failed to demonstrate a risk to the fetus and there are no adequate and well-controlled studies in pregnant women. Category C comprises of drugs in which animal reproduction studies have shown an adverse effect on the fetus and no adequate studies in humans, but the potential benefit may warrant use in pregnancy despite potential risks (table 1). However, in the management of insomnia in the outpatient group, 33.3\% of prescribed drugs were from FDA category N. This category comprises of drugs that are yet to be categorized by FDA and may thus possess higher risk potential.

In the inpatient group, except for treatment of severe anemia, malaria and gastroenteritis in which drugs prescribed were mainly from categories $\mathrm{A}$ and $\mathrm{B}$, prescriptions for other diagnosis were largely drawn from drugs across categories B and C. This could probably be because of the nature of medical conditions that warranted hospitalization and the specialist prescription requirement. Therefore, this group appears to be more prone to higher risk of adverse drug reaction and teratogenicity than the outpatient group.

\section{Limitation}

This study only covered a part of a state in the Northwest region of Nigeria for a period of $6 \mathrm{mo}$, therefore, cannot be extrapolated to other regions of the country. 


\section{CONCLUSION}

With respect to FDA categorization, it can be concluded that drugs prescribed to the pregnant patient in Specialist Hospital, SokotoNigeria, are within reasonable limit of safety. However, as regards management of threatened abortion, insomnia, pre-eclampsia and eclampsia, the use of drugs such as Diazepam, which belong to FDA category $\mathrm{N}$ and are yet to be classified may pose a higher risk of teratogenicity to the fetus and should, therefore, be replaced with alternatives with well-established safety profile. In addition, the percentage of fetal loss, as shown in the fraction of incomplete abortion (18.8\%), was relatively high. Therefore, investigative researches should be conducted in this area in order to unravel the background cause(s) and proffer preventive solution.

\section{ACKNOWLEDGEMENT}

Our gratitude goes to the management of the Sokoto specialist hospital and the entire staffs (clinical and non-clinical) of the Department of Obstetrics and Gynecology. We also want to thank the management of Department of Pharmacology and Toxicology, Usmanu DanFodiyo University, Sokoto, for their co-operation during the course of data collection.

\section{CONFLICT OF INTERESTS}

The authors wish to declare that there was no conflict of interest during and after this research work.

\section{REFERENCES}

1. Sachadeva P, Ptael BG, Patel BK. Drug use in pregnancy; a point to ponder. Indian J Pharm Sci 2009;71:1-7.

2. Briggs GG, Greeman RK, Yaffe SJ. Drugs in pregnancy and lactation: a reference guide to foetal and neonatal risk. $6^{\text {th }}$ Edition. Philadelphia: Lippincott Williams and Wilkins Publishers; 2002. p. 16-8.

3. British National Formulary. Pregnancy 2006;52:760.
4. Sharma R, Kapoor B, Verma U. Drug utilisation pattern during pregnancy in North India. Indian J Med Sci 2006;60:277-87.

5. Koren G, Pastuszak A, Ito S. Drugs in pregnancy. N Eng J Med 1998;338:1135-7.

6. FDA pregnancy categories, compiled by CARE Northwest. Available from: http://depts.washington.edu/druginfo/ Formulary/Pregnancy.pdf. [Last accessed on 20 Mar 2016]

7. World Health Organization Collaborative group on drug use in pregnancy. Medication during pregnancy: an intercontinental cooperative study. Int J Gynand Obs 1992;39:185-96.

8. Tsega B, Shemsu A, Ergetie Z. Assessment of drug utilisation among pregnant women in the university of gondar teaching hospital, Northwest Ethiopia. Glob J Pharm Res 2013;2:1434-40.

9. Abubakar K, Abdulkadir R, Abubakar SB, Jimoh AO, UgwahOguejiofor JC, Danzaki A. Drug utilisation pattern in pregnancy in tertiary Hospital in Sokoto, North West Nigeria. J Health Sci 2014;4:99-104.

10. Ilyaz Md, Roya Rozati, Hafeez F, Tahniyath F, Hasbeen S, Asgfaq Hussein Md. Drug utilisation pattern in pregnancy: a scope for Improvement in the current prescription practices. Int J Pharm 2014;4:156-67.

11. Brown $\mathrm{H}$, Small M. The role of the maternal-fetal medicine in review and prevention of maternal deaths. Perinatology 2012;36:27-30.

12. CDC Fact File on the list of places in the world where malaria transmission occurs. Available from: http://www.cdc.gov/ malaria/travelers/country_table/a.html. [Last accessed on 20 Mar 2016]

\section{How to cite this article}

- $\quad$ Iyabo Adebisi, Tijjani Rabiu Giaze. Drug utilisation pattern in pregnancy amongst inpatient and outpatient groups in a specialist hospital, North-Western Nigeria. Int J Pharm Pharm Sci 2017;9(3):84-87. 